\title{
Criterios \\ Restos neandertales de la Cueva de El Sidrón: una restauración al servicio de la investigación paleontológica
}

Antonio Rosas González' > Javier Fortea Pérez" > Marco de la Rasilla Vives" > Pilar Fernández Colón' > Almudena Hidalgo González' > Elena Lacasa Marquina' > Cayetana Martínez-Maza' > Antonio García Tabernero' > Markus Bastir'

\section{Resumen}

La Cueva de El Sidrón ha deparado la colección de fósiles neandertales más importante de la Peninsula Ibérica. Fruto de las excavaciones realizadas durante las campañas arqueológicas de 2000-2002 se recuperaron cerca de 700 fósiles de la especie Homo neanderthalensis cuya antigüedad se estima entre 60.000 y 40.000 años.

La problemática de conservación y restauración hallada en la Cueva de El Sidrón es una situación frecuente en gran parte de los yacimientos arqueo-paleontológicos en cueva. El entorno sedimentario donde se encuentran enterrados los restos óseos ha dado origen a la formación de concreciones generalizadas sobre la superficie del hueso que, aunque estables, impiden la observación del material para su investigación.

Este articulo destaca la prioridad de llevar a cabo una conservación preventiva en el proyecto de El Sidrón frente a los procesos de restauración.

\section{Palabras clave}

\section{Cueva de El Sidrón}

Borines

Asturias

Conservación

Restauración

Neandertales

Fósiles

Paleoantropología

Concreciones calcáreas

\section{Introducción}

La Cueva de El Sidrón, ubicada en el municipio asturiano de Borines (Piloña), ha deparado la colección de fósiles neandertales más importante de la Península Ibérica. Su hallazgo casual en 1994 sacó a la luz numerosos restos humanos que fueron asignados a la especie Homo neanderthalensis (Rosas y Aguirre, 1999; Prieto et al., 2001; Egocheaga et al., 2000). En las campañas arqueológicas comprendidas entre los años 2000-2002 se extrajeron cerca de setecientos fósiles (Fortea et al., 2003) que actualmente se encuentran en el Museo Nacional de Ciencias Naturales (CSIC) de Madrid en calidad de préstamo de estudio. La cronología estimada se encuentra en el intervalo entre 60.000 y 40.000 años de antigüedad. Previo a la investigación paleoantropológica, hemos analizado el estado de conservación que presenta la colección para efectuar el tratamiento de preservación y restauración más adecuado.

Desde que a mediados del siglo XX en yacimientos como Sterkfontein, Swartkrans, Kromdrai y Environs (Sudáfrica) se recurrió a la dinamita para extraer fósiles de la roca que los contenía, las técnicas de recuperación y conservación de este tipo de materiales han cambiado mucho. Dada la cantidad e interés de los restos paleontológicos que se extraen cada año, poco a poco se ha hecho necesaria la intervención de los restauradores, mediadores entre los hallazgos y el futuro estudio, que se ponen al servicio de la ciencia sin buscar un fin estético, sino el máximo rendimiento de los trabajos de investigación. Para ello, la restauración debe buscar un equilibrio entre el criterio de mínima intervención relativo al uso de consolidantes y productos químicos -en general, no compatibles con los métodos científicos-, y la salvaguarda del fósil.

Una característica frecuente de los restos fósiles es el que aparezcan recubiertos parcial o totalmente por una matriz de variada naturaleza que impide su reconocimiento. Éste es el problema principal, sin fácil solución, en los restos humanos hallados en El Sidrón. De este modo, el objetivo prioritario para la conservación y restauración de esta colección es la eliminación de las concreciones sin dañar la fina y sensible capa cortical ósea del fósil, intimamente unida al sedimento. Dicha superficie debe mantenerse intacta por la valiosa información que contiene, la cual será analizada en posteriores estudios tafonómicos y microestructurales.

\section{Estado de conservación de la colección de neandertales de El Sidrón}

Los fósiles de El Sidrón presentan un estado de conservación precario, consecuencia de las alteraciones relativas a la fosilización, tafonomía y condiciones ambientales a las que estuvieron sometidos. Su problemática de conservación y restauración es frecuente en gran parte de los yacimientos arqueo-paleontológicos en cueva. 
' Departamento de Paleobiología. Museo Nacional de Ciencias Naturales. CSIC. Madrid

"Área de Prehistoria. Universidad de Oviedo
1. Ejemplo de brecha de huesos humanos procedente del yacimiento de El Sidrón (Asturias). Se aprecia cómo los restos óseos están recubiertos por sedimentos carbonatados y endurecidos. La retirada de esta matriz envolvente sin causar daño a los materiales fósiles constituye un auténtico reto en la restauración y conservación del patrimonio arqueo-paleontológico.
Aunque los parámetros ambientales y de conservación suelen ser comunes en este tipo de entorno, existen diferencias según la morfología del sistema kárstico (Laborde, 1986). En el caso de El Sidrón, el yacimiento fue localizado en una estrecha galería del interior donde la temperatura se mantiene sin fluctuaciones de importancia y el índice de humedad relativa debió de estar próximo a la saturación. La matriz en la que fueron hallados los restos es de naturaleza arcillo-arenosa con variaciones de color y textura que explicaremos más adelante.

Estos factores ambientales desencadenaron los siguientes procesos: la fosilización, que reemplazó los compuestos orgánicos por otros de naturaleza mineral, y la carbonatación del sedimento sobre la superficie fósil, que dio lugar a lo que denominamos concreciones calcáreas que, aunque en sí mismas no perjudican al resto paleontológico, impiden su legibilidad. En el caso del material de El Sidrón hemos diferenciado cinco tipos de concreciones carbonáticas aisladas o dispuestas de forma laminar; es decir, en una misma pieza se pueden distinguir uno o varios tipos de concreciones:

> Barro endurecido, de color pardo grisáceo y sonido cristalino, localizado en las capas más externas. El 13\% del material presenta este tipo de concreción formada por arena carbonatada con bioturbación y materiales detríticos como gasterópodos y granos de cuarzo.

$>$ Costras laminadas intermitentes, presentes en el 1,5\% de los fósiles, de color pardo y gran dureza, por encima de las cuales se observa el barro endurecido. Se diferencian dos clases: una de grano grueso con gasterópodos muy adaptada al hueso, color anaranjado y quizá contemporánea a la deposición del fósil; y otra de grano más fino con bioturbación y color grisáceo, que recubre e incluso rellena la estructura ósea y cuya formación podría ser posterior a la deposición del hueso.

$>$ Formas globulíticas, de tono pardo, simulan cristales y se hallan en zonas aisladas, en un 3\% del material.

> Microbrecha de huesos, de color ocre pálido y muy deleznable, forma un conglomerado con el sedimento. Esta concreción se ha observado en el $4 \%$ de las piezas.

$>$ Concreciones muy adheridas a la superficie fósil, de color ocre anaranjado y escaso espesor (0,5- $2 \mathrm{~mm})$, muy duras e infiltradas en grietas y fisuras, presentes el $50 \%$ de los fósiles.

Además, hay que añadir que el $49 \%$ de los fósiles no muestran ningún tipo de concreción, tan sólo los recubre un fino polvo anaranjado o suciedad superficial.

Otro agente importante en la degradación del material óseo es su propia naturaleza físico-química, ya que sus propiedades higroscópicas y anisotrópicas, ligadas a los cambios de humedad relativa

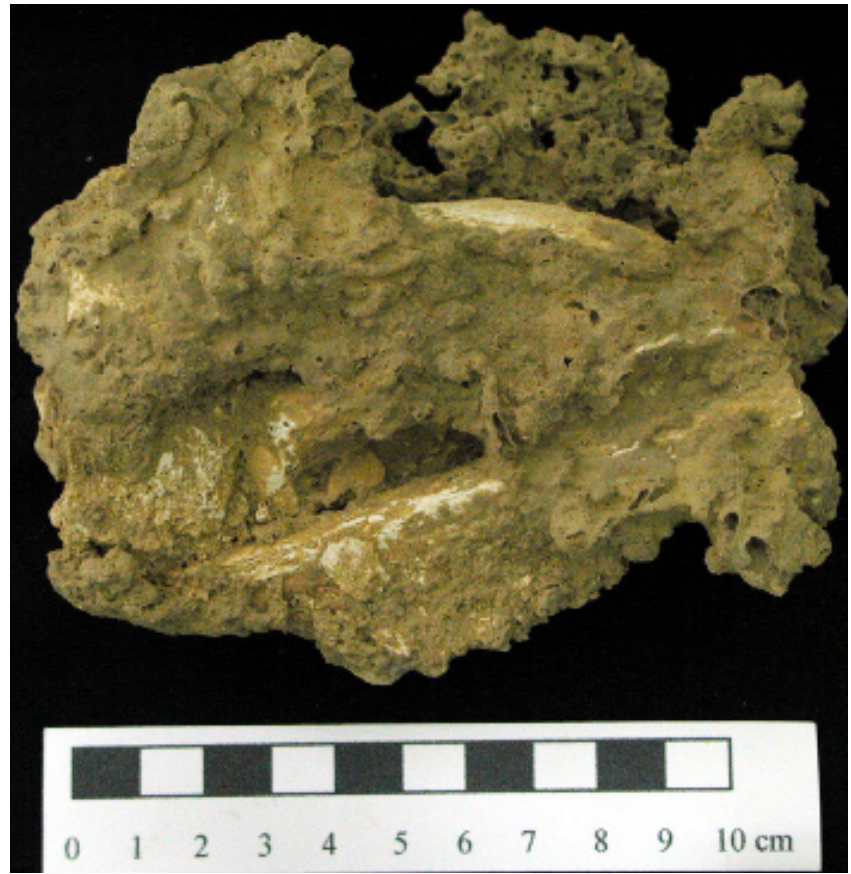

y temperatura, dan lugar a procesos de expansión/contracción de la estructura del hueso.

La combinación de todos estos factores hace que los fósiles de El Sidrón se encuentren altamente deshidratados, deleznables y con múltiples microfisuras que en algunos casos han llegado a convertirse en grietas y fracturas. Aunque esta circunstancia es común a toda la colección, dado el elevado número de fósiles que la componen, podemos diferenciar tres niveles en función del grado de deterioro físico: el 27\% del material presenta una acusada fragmentación que imposibilita su estudio morfológico; el 11\% se encuentra fragmentado con posibilidad de reconstrucción; y el $62 \%$ conserva totalmente su morfología.

\section{Material y métodos}

Tras comprobar el frágil estado de conservación que presentan los fósiles de El Sidrón, se concretaron los tratamientos más convenientes para su estabilización estructural y para la retirada total de las concreciones carbonáticas que los recubren sin dañar su capa cortical. Con este fin, hemos procedido a la intervención del material en varias fases:

\section{Documentación previa}

Para orientar la limpieza y poder actuar de forma rápida y precisa, se radiografiaron aquellos restos sin una clara diferenciación entre la matriz calcárea que los recubría y la superficie del fósil. 


\begin{tabular}{|c|}
\hline Criterios \\
\hline $\begin{array}{l}\text { Restos neandertales de la } \\
\text { Cueva de El Sidrón: una } \\
\text { restauración al servicio } \\
\text { de la investigación } \\
\text { paleontológica }\end{array}$ \\
\hline $\begin{array}{l}\text { Antonio Rosas González } \\
\text { y otros }\end{array}$ \\
\hline
\end{tabular}

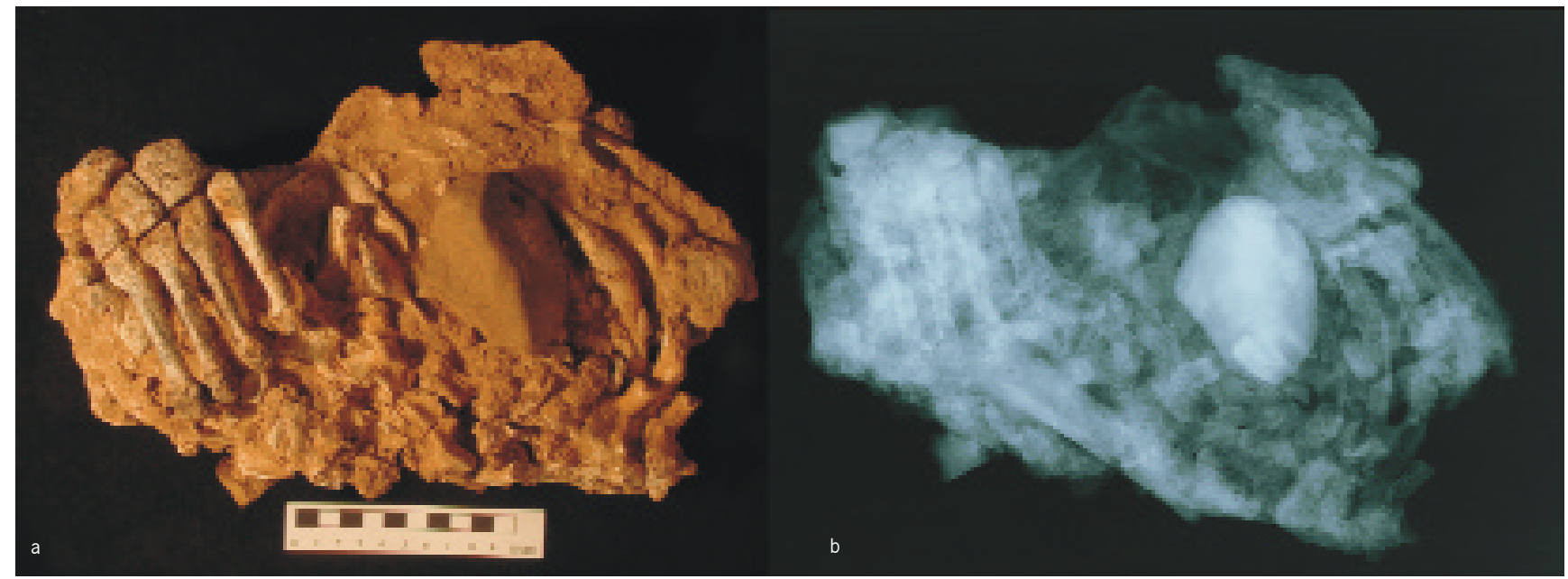

\section{Intervención de limpieza}

Teniendo en cuenta la irreversibilidad que implica el tratamiento de limpieza, es esencial desarrollar una metodología que asegure un control total sobre la materia que estamos eliminando sin dañar la superficie fósil. Para lograr una limpieza segura y eficaz, hemos ensayado diferentes medios seleccionando los más adecuados conforme a la naturaleza de cada concreción. De esta manera, se concluyó que la multi-herramienta digital de alta velocidad Dremel modelo 398 era más apropiada para la retirada de las costras de barro endurecido más externas, mientras que utensilios manuales como bisturies y material de dentista combinados con disolventes eran más eficaces sobre las concreciones globuliticas, laminadas y las superficiales muy adheridas al fósil.

La experiencia en anteriores trabajos de limpieza de molares pertenecientes al material paleontológico del yacimiento de Pinilla del Valle (Madrid), de características similares a la colección de El Sidrón, demostró la efectividad del vibroincisor para la eliminación de las concreciones menos adheridas. Por el contrario, se descartó el uso de cubetas de ultrasonidos por la fragilidad y numerosas fisuras que presentan la mayoría de los restos, así como el empleo de ácidos, dado que su grado de penetración y permanencia es difícil de controlar, pudiendo interactuar con el material fósil y alterarlo (Landucci et al., 2003).

Aunque no ha habido posibilidad de ensayarlo sobre el material de El Sidrón, una opción no descartada es la aplicación del láser para eliminar las concreciones calcáreas adheridas a la cortical del fósil, capa donde se incrementa el riesgo de daño durante la limpieza mecánica. Recientemente se han publicado artículos sobre el uso combinado del láser ( $\mathrm{Nd}$ :Yag laser radiation) con métodos convencionales de limpieza sobre restos paleontológicos con resultados satisfactorios. Sin embargo, este instrumental tiene un coste elevado y el proceso es lento (Landucci et al., 2003).
2. a: Acumulación de restos humanos original englobados en una matriz carbonatada. Espécimen 437 de El Sidrón. b: Radiografia de la misma acumulación donde se pueden distinguir diferentes elementos anatómicos incluidos en la matriz sedimentaria.

\section{Medidas de conservación}

Como ya se mencionó, nos encontramos ante un problema complejo debido al delicado estado de los fósiles y a la dura concreción que los recubre, que en ocasiones se convierte en su único soporte estructural. Puesto que un criterio museistico de conservación y restauración requiere del uso de productos químicos y tratamientos que a priori son incompatibles con los métodos analiticos de investigación, la colección de El Sidrón precisa una metodología de actuación alternativa. De esta forma, optamos por una conservación preventiva minuciosa por medio del control de los factores ambientales y de un almacenamiento adecuado para crear un microclima óptimo que frene el deterioro de los fósiles (Collins, 1995). Mediante el gel acondicionado Art Sorb, mantenemos la humedad relativa al 50\%, mientras que la temperatura de $20^{\circ} \mathrm{C}$ se sostiene gracias al aislamiento térmico que el poliestireno expandido proporciona al armario que alberga la colección. Para la disposición individual de estos restos paleontológicos se recurrió a cajas inertes de polietileno con espuma ethafoam en su interior para evitar los nocivos efectos que vibraciones accidentales, roces o entrada de polvo pudieran provocar.

Previa consulta con los investigadores, el uso de resinas acrílicas estables y solubles es inevitable en aquellos casos que requieren devolverle al fósil su estabilidad estructural, aplicándose en mínimas concentraciones mediante procesos reversibles de consolidación y adhesión. La selección de un producto inadecuado o mal empleado puede invalidar los resultados de muchas investigaciones, como es el caso de la emulsión acrílica Primal AC33, muy utilizada en materiales paleontológicos y cuyo envejecimiento deja una película plástica que resulta insoluble tanto en el interior como en la superficie del fósil. Para la colección de El Sidrón, hemos recurrido a la resina acrílica Paraloid B-72 al 2\% en acetona para consolidar y al $10 \%$ para las adhesiones (Collins, 1995). En los casos en que se necesitó una consolidación más profunda, se uti- 

técnicas de reconstrucción virtual de restos fósiles puede ser de gran utilidad en la restauración y conservación del patrimonio paleontológico.

lizó además la resina polivinílica Mowital al 5\% en alcohol etílico, de características similares al Paraloid B-72. Estas resinas se aplican por impregnación con pincel para controlar mejor la cantidad y grado de penetración del consolidante empleado, rechazando por excesiva e imprecisa la consolidación al vacio y por inmersión.

\section{Conclusión}

En este trabajo presentamos la colección más importante de fósiles neandertales de la Península Ibérica procedentes de la cueva de El Sidrón (Borines, Asturias). El estudio de este material requiere un primer análisis desde el punto de vista de la restauración y la conservación. En este sentido, se han diferenciado cinco tipos de concreciones calcáreas y tres niveles de conservación variables que han determinado el criterio de intervención. Los primeros resultados obtenidos constatan la eficacia de la metodología aplicada y se plantea la posibilidad de utilizar nuevas técnicas para la preservación del material reforzando el compromiso con la investigación.

\section{Agradecimientos}

Expresamos nuestra gratitud a todas las personas que de un modo u otro han contribuido a la excavación y recuperación del material aqui estudiado. Este trabajo se inscribe en el desarrollo científico del Convenio Consejería de Cultura del Principado de Asturias/Universidad de Oviedo (CN-00-184-D3 y CN-01-132,133,134-B1) y del Plan I+D+1 Concertada (Ficyt/Consejería de Cultura: FC-02-PCSPV01-27). Algunos de los aspectos aquí incluidos son objeto de estudio del proyecto BOS2003-01531 del MEC.

\section{Bibliografía}

COLLINS, C. The care and conservation of palaeontological material. ButterworthHeinemann, 1995.

EGOCHEAGA, J.E.; TRABAZO, R; RODRIGUEZ, L.; CABO, L.L.; SIERRA, M.J. Avance sobre el descubrimiento, características del yacimiento y estudio paleoantropológico de los restos óseos de homínidos mesopleistocénicos de la Cueva de Sidrón (Piloña, Asturias). Bol. Cien.Nat. RIDEA, 46, 2000, p. 219-263

ESCUDERO, C.; ROSSELLÓ, M. Conservación de materiales en excavaciones arqueológicas. Museo Arqueológico de Valladolid, 1988

FORTEA, J.; DE LA RASILLA, M.; MARTINEZ, E.; SANCHEZ-MORAL, S.; CA ÑAVERAS, J. C.; CUEZVA, S.; ROSAS, A.; SOLER, V.; JULIÁ, R.; DE TORRES, T.; ORTIZ, J. E.; CASTRO, J.; BADAL, E.; ALTUNA, J.; ALONSO, J. La cueva de El Sidrón (Borines, Piloña, Asturias): primeros resultados. Estudios geológicos, n ${ }^{\circ} 59$ (1-4), 2003, p. 159-180

LABORDE, A. Conservación y restauración en yacimientos prehistóricos (restos óseos, madera y piedra). Cahier noir, $n^{\circ} 3$ Centre de Recerques Paleo-eco-socials. Gerona, 1986

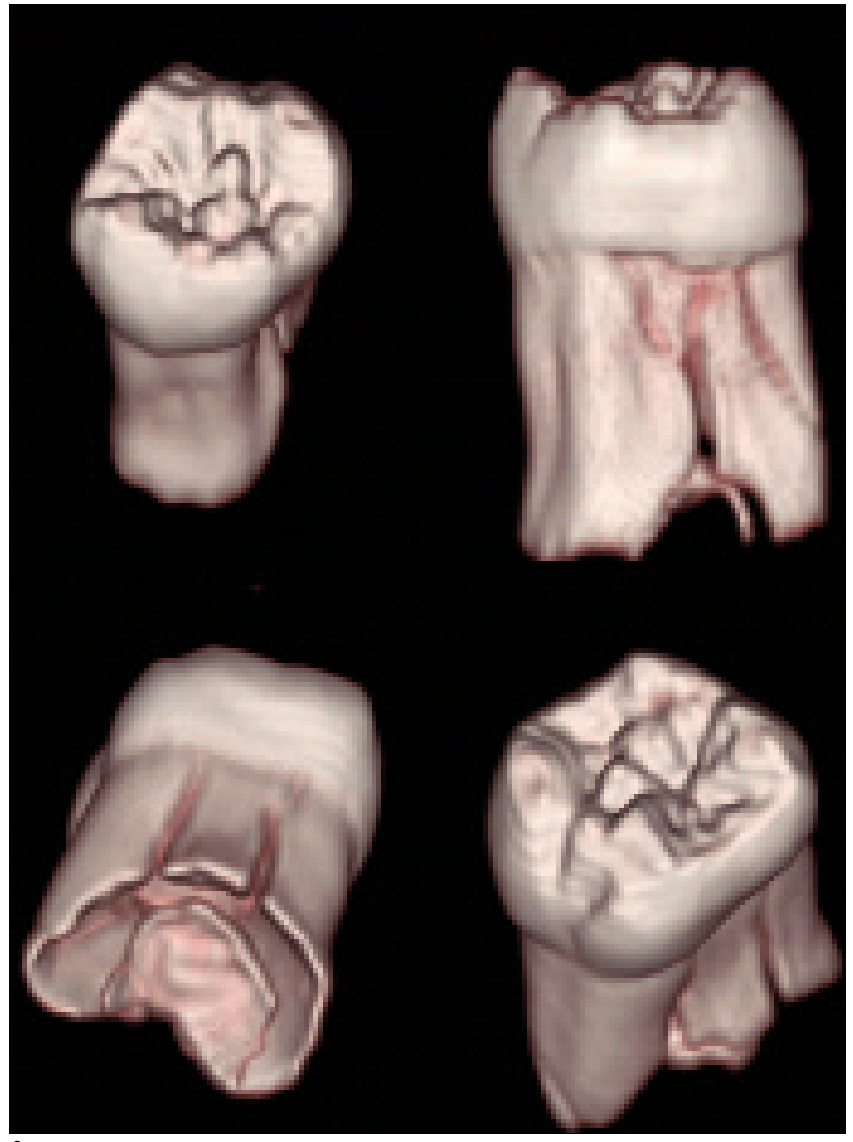

LANDUCCI, F.; PECCHIONI, E.; TORRE, D.; MAZZA, P.; PINI, R.; SIANO, S.; SALIMBENI, R. Toward an optimised laser cleaning procedure to treat important paleontological specimens. Journal of cultural heritage [en linea], v.4, 2003, p. 106-110

LANDUCCI, F.; PINI, R.; SIANO, S.; SALIMBENI, R.; PECCHIONI, E. Laser cleaning of fossil vertebrates: a preliminary report. Journal of Cultural Heritage [en línea], v.1, 2000, p. 263-267

PORTO, Y. Medidas urgentes de conservación en intervenciones arqueológicas. Laboratorio de Arqueoloxía e Formas Culturais. Universidad de Santiago de Compostela, 2000

PRIETO, J.L.; ABENEA, J.; MONTES, R.; SANGUINO, J. Y MUÑOZ, E. Hallazgos antropológicos y arqueológicos en el complejo kárstico de El Sidrón (Vallobal, Infiesto, Concejo de Piloña, Asturias). Munibe, 53, 2001, p. 19-29

ROSAS, A.; AGUIRRE, E. Restos humanos neandertales de la Cueva del Sidrón, Piloña, Asturias. Nota preliminar. Estudios Geológicos, 55, 1999, p. 181-190

ROSAS, A.; AGUIRRE, E. Neandertales de la Cueva del Sidrón. Investigación y Ciencia, $n^{\circ} 289,2000$, p. $28-29$

ROSAS, A.; FORTEA, J.; DE LA RASILLA, M.; BASTIR, M.; MARTÍNEZ-MAZA, C. Neanderthals from El Sidrón cave (Asturias, Spain). Presentation of a new sample. American Journal of Physical Anthropology, $n^{\circ} 123$ S 38, 2003, p. 169 